\title{
LES ANTI-INFLAMMATOIRES NON STEROIDIENS
}

RÉSUMÉ : Nous avons à notre disposition un grand nombre de molécules faisant partie de la classe des anti-inflammatoires non stéroîdiens. Ces différentes molécules rendent de grands services aux patients souffrant de pathologies articulaires : on les cite cependant - injustement - plus par certains de leurs effets secondaires, notamment gastro-intestinaux et rénaux, que par le bénéfice qu'elles apportent. La notion de susceptibilité individuelle est admise depuis peu mais diffeile a prouver scientifiquement quoique cadrant parfaitement avec la pratique clinique courante. Elle permet des lors des alternatives qui permettent de garder à cette classe de médications une bonne efficacité et une bonne tolérance.

Des progrès substantiels sont réalisés qui vont modifier nos prescriptions dans un futur proche : inlibition selective des Cox 2, nouveau dérivé bloquant également les leucotriènes. C'est l'utilisation raisonnable et raisonnée des anti-inflammatoires qui permettra à ces molécules de garder leur rôle fondamental dans le contrôle de la réaction inflammatoire principalement rhumatologique

\section{HistoriQue et CLASSIFICATION}

Depuis l'introduction de l'indométhacine en 1963, la littérature scientifique a fréquemment fait état de ce que les composés non stéroïdiens étaient doués de propriétés anti-inflammatoires.

Si l'on tient compte de l'efficacité clinique: prouvée des deux acides carboxyliques présents dans l'acide acétylsalicylique et dans l'indométhacine dans le traitement de la maladie inflammatoire chez l'homme, il n'est peut-être pas surprenant que la grande majorité des substances non stéroïdiennes développées par la suite et douées d'activité anti-inflammatoire soient des acides organiques simples. Si 1'on a signalé des propriétés anti-inflammatoires au niveau de quelques molécules non acides, les études de la relation entre structure et activité ont généralement penché en faveur de molécules capables d'atteindre une configuration planaire contenant un noyau aromatique et possédant une fonction acide. Le tableau I représente une classification schématique des anti-inflammatoires non stéroïdiens.

La première famille, la plus importante, est la classe des acides carboxyliques, classe possédant plusieurs sous-classes, et c'est dans cette famille que 1'on trouve la majorité des antiinflammatoires non stérö̈diens utilisés. La deuxième famille est celle des dérivés énoliques qui, très longtemps, ne s'est composée que de la classe des pyrazolones; en 1971, aux Etats-Unis, une nouvelle classe, celle des oxicams, a été

(1) Agrégé, Chef de Service, Université de Liège ,Service de Rhumatologie.
TABLEAU I. CLASSIFICATION DES ANTI-INFLAMMATOIRES NON STÉROIIDIENS

\begin{tabular}{|c|c|c|}
\hline Acides carboxiliques & $\begin{array}{l}\text { Acides salicyliques } \\
\text { et esters } \\
\text { Acides acétiques } \\
\text { phenyjlacétiques: } \\
\text { carbo- et hétero- } \\
\text { cycliques } \\
\text { Acites propioniques } \\
\text { Acides fenamiques }\end{array}$ & $\begin{array}{l}\text { Acide accétylsalicylique, } \\
\text { ditlunisal, benorylate } \\
\text { Diclofénac, alclofénac } \\
\text { Indométhacine, sulindac, } \\
\text { tolnétine, proglimélacine, } \\
\text { etodolac } \\
\text { lbuproféne, naproxene, } \\
\text { flurbuproféne, fénoprofène, } \\
\text { kétoproféne, } \\
\text { acide tiaprofénique } \\
\text { Acides flulénamique, } \\
\text { nifluminque }\end{array}$ \\
\hline Acides ćnoliques & $\begin{array}{l}\text { Prazolones } \\
\text { Oxicams }\end{array}$ & $\begin{array}{l}\text { Oxyphenbutazone, } \\
\text { phénylbutazone } \\
\text { Piroxicam, ténoxicam, } \\
\text { méloxicam }\end{array}$ \\
\hline No & & Bufexamac, nabumétone \\
\hline Sulfoanilide & & Nimésulide : \\
\hline
\end{tabular}

développée permettant la commercialisation en $1982 \mathrm{du}$ premier oxicam sur le marché, le piroxicam. La troisième classe est celle des composés non acides représentés essentiellement par le bufexamac et la nabumétone non commercialisée. Enfin, il existe une nouvelle famille, toujours acide mais dépourvue d'acide carboxylique, celle présentant un radical sulfoanilide dont il n'existe à ce jour qu'un représentant en voie de commercialisation immédiate : le nimésulide.

Leur appartenance à des classes physico-chimiques non strictement superposables détermine également des variations dans leurs propriétés physico-chimiques chez l'homme : caractère plus ou moins liposoluble pouvant influencer une plus grande fréquence d'effets secondaires touchant certaines sphères cognitives du système nerveux central, pourcentage de forme ionisée par rapport à la forme non ionisée influençant la distribution des médicaments dans les tissus enflammés (les anti-inflammatoires acides sont séquestrés préférentiellement dans le tissu synovial), existence de prodrogue comme le sulindac traditionnellement considéré comme n'interférant pas avec les prostaglandines rénales.

Une des caractéristiques les plus évidentes concerne des demi-vies différentes (tableau II) permettant de les classer en molécules à courte demi-vie (moins de $6 \mathrm{~h}$ ) et en molécules ayant une longue demi-vie (plus de $10 \mathrm{~h}$ ). Sur un plan strictement pharmacologique, parce que l'équilibre plasmatique n'est atteint qu'après une période équivalente à 3 à 5 fois $1 \mathrm{la}$ demi-vie, les anti-inflammatoires à longue demi-vie n'attei- 


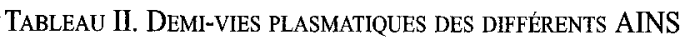

\begin{tabular}{|c|c|}
\hline Médicanent & Demi-vie (h) \\
\hline Counte & \\
\hline Aspirine & 0,25 \\
\hline Diclofénac & 11 \\
\hline Etodolac & 3 \\
\hline Ftaoprofene & 2,5 \\
\hline Acide flufénamique & 1,4 \\
\hline Flubbiprofene & 3,8 \\
\hline lbuprofene. & 2,1 \\
\hline Indonethacine & 4,6 \\
\hline Ketoprofene & 1,8 \\
\hline Nimesulide & 4. \\
\hline Longue: & \\
\hline Difluisal & 13 \\
\hline Nabirnétone & 26 \\
\hline Naproxene & 14 \\
\hline Plienylbutazone & 68 \\
\hline Piroxican & 57 \\
\hline Sulindac & 14 \\
\hline Tenoxicam & 60 \\
\hline Méloxican & 20 \\
\hline
\end{tabular}

gnent pas aussi rapidement leur plateau de concentration plasmatique que les anti-inflammatoires à demi-vie courte. Il n'est cependant pas du tout démontré que cela se traduise par une perte de rapidité d'action clinique.

A côté de la demi-vie qui peut être un argument à retenir lorsque l'on doit choisir un antiinflammatoire non stéroïdien pour une situation aiguë (un jour ou deux de traitement), ou pour une situation chronique, il existe d'autres propriétés comme leur interaction ou non avec les anticoagulants oraux qui peuvent faire choisir l'une ou l'autre molécule dans cette situation particulière. Leur liaison à l'albumine est forte (plus de 95\%) et les variations de fraction libre d'une classe à l'autre n'ont guère de répercussions cliniques pratiques.

\section{MÉCANisme D'ACTION}

En 1971, il a été démontré que 1'aspirine et l'indométhacine inhibaient la cyclo-oxygénase (Cox) d'homogénat de poumon de cobaye, de plaquettes humaines et, in vivo, au niveau de la rate du chien, prévenant la synthèse des prostaglandines et molécules dérivées (prostanoïdes). L'inhibition de cette production de prostaglandine a dès lors été proposée comme étant le mécanisme commun d'action de l'ensemble des anti-inflammatoires non stéroïdiens. Bien que le mécanisme moléculaire puisse être un peu différent de molécules à molécules, ces données n'ont pas de répercussion pratique : on peut simplement garder à l'esprit le parallélisme existant entre l'efficacité et l'inhibition de la molécule in vitro et son pouvoir anti-inflammatoire in vivo. L'inhibition de la cyclo-oxygénase explique également directement les effets secondaires classiquement attribués à cette molécule : ulcération digestive essentiellement de la muqueuse gastrique puisque la prostacycline en est un important cytoprotecteur, inhibition des prostaglandines rénales perturbant la régulation physiologique de cet organe.

\section{Progrès Récents CONCERNANT Les} ANTI-INFLAMMATOIRES NON STÉROÏDIENS

Trois grands progrès sont susceptibles de modifier, dans les prochaines années, notre façon d'envisager le traitement des maladies inflammatoires par anti-inflammatoires non stéroïdiens: l'inhibition sélective de la cyclo-oxygénase inductible, l'inhibition de la voie de la lipoxygénase et une modification galénique des molécules actuelles.

\section{CYCLO-OXYGÉNASE INDUCTIBLE.}

Le premier est la découverte il y a 5 ans d'une seconde cyclo-oxygénase appelée Cox 2 par opposition à la cyclo-oxygénase classique appelée Cox 1 et qui est une cyclo-oxygénase inductible par opposition à la type 1 qui est constitutionnelle. L'expérience, déterminante, avait montré que le lipopolysaccharide bactérien augmentait la synthèse de prostaglandines à la fois chez le monocyte humain in vitro et chez le macrophage péritonéal murin in vivo. Cette augmentation était inhibée par la dexaméthasone et associée avec une synthèse de novo d'une nouvelle protéine de type cyclo-oxygénase. L'isoforme inductible (Cox 2) a été indentifiée un an plus tard : elle est encodée par des gènes différents de l'enzyme constitutionnelle et sa séquence d'acides aminés ne montre qu'une homologie de $60 \%$ avec Cox 1 . Les deux enzymes ont cependant un poids moléculaire voisin de $70 \mathrm{kDa}$ et des récepteurs identiques tant pour leur substrat naturel que pour l'inhibition par les anti-inflammatoires non stéroïdiens.

Le profil pharmacologique de Cox 1 et de Cox 2 est également différent de sorte qu'il devient possible de développer de nouveaux anti-inflammatoires non stéroïdiens qui sont des inhibiteurs spécifiques de Cox 2 ou qui inhibent très préférentiellement Cox 2 en laissant intact Cox 1. Dans cette optique, les effets secondaires classiquement attribués à l'inhibition de la cyclo-oxygénase constitutionnelle devraient être considérablement réduits par 1'utilisation de ces nouvelles molécules. Le nimésulide et le méloxicam sont deux molécules présentant un profil préférentiel de type Cox 2 . 


\section{INHIBITION DES VOIES DE LA LIPOXYGÉNASE}

On sait depuis longtemps que l'inhibition de la cyclo-oxygénase ne représente qu'une approche partielle de l'inhibition des mécanismes inflammatoires puisqu'elle laisse intacte la possibilité de génération de différents médiateurs phlogogènes par les voies de la lipo-oxygénase. C'est d'ailleurs comme cela que l'on explique le déclenchement de crises d'asthme chez les patients prenant un anti-inflammatoire non stéroïdien, le blocage de la cyclo-oxygénase favorisant 1'orientation des dérivés phospholipidiques vers la voie de la lipoxygénase. Plusieurs courants de recherche visent donc à essayer de mettre au point des molécules qui possèdent la capacité d'inhiber aussi la voie de la lipoxygénase : le zyleuton, le tenidap sont deux nouvelles molécules présentant des propriétés de ce type et susceptibles dès lors de constituer de nouvelles approches du traitement de l'inflammation.

\section{PROGRÈS GALÉNIQUES}

A côté de modifications fondamentales des propriétés des molécules, existe tout un courant de recherche visant à modifier sa biodisponibilité et à tenter de réduire l'incidence d'effets secondaires : ainsi sont nées de multiples formes entériques résorbées préférentiellement le long du tractus digestif ou encore l'association de l'anti-inflammatoire à un vecteur (l'association piroxicam - bêta-cyclodextrine) qui en permet la résorption gastrique immédiate.

\section{INDICATIONS DES ANTI-INFLAMMATOIRES NON STÉROÏDIENS}

Les pathologies articulaires et abarticulaires représentent les indications classiques des antiinflammatoires non stéroïdiens : arthrite, arthrose, pathologie tendineuse, traumatologie sportive... On leur découvre également d'autres indications comme dans la douleur postopératoire, les douleurs dentaires, les dysménorrhées pour ne citer que les exemples les plus utilisés. Une dernière notion importante à connaître, mais qui ne fait l'objet d'aucune étude scientifique l'ayant démontré de manière formelle, est la notion de susceptibilité individuelle tant dans la réponse clinique à l'anti-inflammatoire que dans la genèse d'un effet secondaire. En pratique donc, l'inefficacité perçue par le patient, ou l'énoncé d'un effet secondaire rapidement apparu lui faisant cesser la médication, ne condamne en rien le recours à d'autres molécules d'une même sous-classe peut-être, mais surtout d'une sous-classe différente. Il faut dès lors tâtonner afin de déterminer la molécule qui convient le mieux au patient.
Enfin, et particulièrement dans ce domaine fréquent qu'est la douleur arthrosique, la prescription d'anti-inflammatoires non stéroïdiens reste parfaitement justifiée lors des crises (micro-inflammatoires locales) qui émaillent le parcours du patient arthrosique. Il y a 10 ans environ, tout un courant de recherche a voulu étudier les interactions potentielles entre antiinflammatoires non stéroïdiens et matrice cartilagineuse. Plusieurs travaux in vitro, essentiellement chez l'animal dans des modèles variés d'arthrose, ont montré que certaines molécules pouvaient avoir un effet délétère sur les capacités de régénération cartilagineuse. Il faut remarquer que les molécules incriminées n'étaient jamais les mêmes selon le modèle animal étudié. L'extrapolation de ces données en pathologie humaine est la résultante d'un amalgame peu scientifique, et les études réalisées en culture humaine de cartilage n'ont jamais pu reproduire les données animales ni par ailleurs, inversement, identifier une molécule qui pourrait se targuer du qualificatif de chondroprotection. Ceci n'exclut pas la possibilité d'interaction in vivo entre anti-inflammatoires non stérö̈diens et fonction chondrocytaire (notamment par le fait que l'inhibition des prostaglandines locales puisse amplifier la production d'interleukine 1), mais ces notions sont du domaine de la recherche et ne peuvent jeter le discrédit sur une classe de médicaments irremplaçables dans le contrôle de la poussée arthrosique.

\section{RÉFÉRENCE}

Brooks PM, Day RO.- Nonsteroidal antiinflammatory drugs - differences and similarities. $N$ Engl $J$ Med, 1991, 324, 1716-1725. 